\title{
Selection of Tau Leptons with the CDF II Trigger System
}

\author{
S.Baroiant $^{\mathrm{a}}$, M.Chertok ${ }^{\mathrm{a}}$, M.Goncharov ${ }^{\mathrm{b}}$, T.Kamon ${ }^{\mathrm{b}}$, V.Khotilovich ${ }^{\mathrm{b}}$, R.Lander ${ }^{\mathrm{a}}$, P.Murat ${ }^{\mathrm{c}}$,

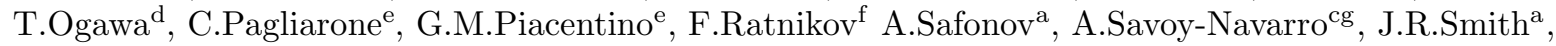 \\ D.Toback ${ }^{\mathrm{b}}$, S.Tourneur ${ }^{\mathrm{cg}}$, E.Vataga ${ }^{\mathrm{e} *}$ \\ a Department of Physics,University of California, Davis, CA 95616, USA \\ ${ }^{\mathrm{b}}$ Department of Physics, Texas A\&M University, College Station, TX 77843, USA \\ ${ }^{\mathrm{c}}$ Fermi National Accelerator Laboratory, Batavia, IL 60510, USA \\ ${ }^{\mathrm{d}}$ Department of Physics, Waseda University, Tokyo 169, Japan \\ eIstituto Nazionale di Fisica Nucleare, I56100 Pisa, Italy \\ ${ }^{\mathrm{f}}$ Department of Physics and Astronomy, Rutgers, the State University of New Jersey, Piscataway, NJ \\ 08854, USA
}

g LPNHE Universites de Paris 6 et 7/IN2P3-CNRS, Tour 33, RdC 4, Place Jussieu, 75252 Paris Cedex 05, France

In Run II of the CDF experiment, traditional dilepton triggers are enriched by lepton $(e$ or $\mu)$ plus track, di- $\tau$ and $\tau$ plus missing transverse energy triggers at Level-3 dedicated to physical processes including tau leptons. We describe these triggers, along with their physics motivations, implementation and cross-sections and report on their initial performance.

\section{Introduction}

Run II of the Tevatron produces $p \bar{p}$ interactions with an instantaneous luminosity about $10^{32} \mathrm{~cm}^{-2} \mathrm{~s}^{-1}$. The CDF Detector trigger system 1 decreases the resulting initial event rate of $2.5 \mathrm{MHz}$ down to $70 \mathrm{~Hz}$ for data storage and offline analysis, with the goal of efficient extraction of interesting physics events from the large minimum bias background. One important subset of these physics signatures includes $\tau$ leptons. Events with $\tau$ 's are important for the study of various Standard Model (SM) processes such as $Z \rightarrow \tau \tau, t \bar{t}$ with $W \rightarrow \tau \nu_{\tau}, H \rightarrow \tau \tau$ and also to extend the reach of searches for physics beyond the SM. These physics goals drive us to look for $\tau$-like objects starting with Level-3 of the trigger, and our implementation selects events with two

\footnotetext{
*Corresponding author. Tel.: +39-050-2214415; fax: +39-
} 050-2214317; e-mail: Elena.Vataga@pi.infn.it leptons in the final state: $e \tau_{h}, \mu \tau_{h}, \tau_{h} \tau_{h}$ and also $\tau_{h} \nu$, as well as $e e, e \mu$ and $\mu \mu$. With " $\tau_{h}$ " we indicate hadronically decaying $\tau$ 's, while the $e$ or $\mu$ can be either produced directly or through a leptonic decay of the $\tau$.

These tau triggers benefit from the upgraded CDF trigger system, in particular the tracking processor at Level-1 (L1) and the refined tracking information available at Level-2 (L2) [12].

\section{Implementation of the $\tau$ Triggers}

\section{1. $\tau$ reconstruction}

Taus promptly decay in leptonic or hadronic (65\%) modes with at least one $\nu$ in the final state. Hadronic $\tau$ decays have the distinct signature of a narrow isolated jet with low multiplicity (1 or 3 prongs) and low visible mass $\left(<M_{\tau}\right)$. The basis for our triggers, therefore, is a $\tau$-cone algorithm for the reconstruction of these decays. To 
build the $\tau$-cone object, we start with a narrow calorimeter cluster, above a suitable $E_{T}$ threshold, matched to a seed track with momentum above a $P_{T}$ cut. The region within an angle $\Theta_{\text {Sig }}$ from the seed track direction is used to define a cone of tracks to be associated with the $\tau$. The region between $\Theta_{\text {Sig }}$ and $\Theta_{I s o}$ defines the isolation cone: we require that no tracks with $P_{T}$ higher than a fixed low threshold be found in this region. At the Level-3 (L3) of the trigger, $\Theta_{\text {Sig }}$ and $\Theta_{\text {Iso }}$ have values of $10^{\circ}$, and $30^{\circ}$, respectively, and are determined in 3 dimensions.

We have implemented 4 different triggers for physics processes with $\tau$ 's in the final state, installed in the CDF trigger tables in January 2002. Below we describe their main features.

\subsection{Electron plus track, Muon plus track}

The selection for the $e$ plus track trigger starts with L1 and L2 requirements of a single EM tower with $E_{T}>8 \mathrm{GeV}$ with an associated XFT track with $P_{T}>8 \mathrm{GeV} / c$ and a second track with $P_{T}>5 \mathrm{GeV} / c$. At L3, these conditions are refined and charged track isolation around the reconstructed (2nd) track is imposed. The current average cross-section for this L3 trigger is $\sim 29$ nb. For the muon plus track trigger we require a muon stub matched at L1 to an XFT track with $P_{T}>4 G e V / c$, with an increased track threshold of $8 \mathrm{GeV} / c$ at L2. The "track" requirements are identical to those for the electron plus track trigger. The current average cross section for this trigger is $\sim 16 \mathrm{nb}$.

\subsection{Di- $\tau, \tau$ plus Missing Transverse Energy}

At L1 the di- $\tau$ trigger requires 2 calorimeter towers with $E_{T}>5 \mathrm{GeV}$ and 2 matching XFT tracks with $P_{T}>6 \mathrm{GeV} / c$, separated by an angle of $\phi>30^{\circ}$. L2 requires cluster $E_{T}>10 \mathrm{GeV}$ and imposes track isolation. At L3, using the full reconstruction code, $2 \tau$ candidates with seed track $P_{T}>6 \mathrm{GeV} / c$, and originating from the same vertex: $|\Delta Z|<10 \mathrm{~cm}$, are required. The L3 cross section for this trigger is $\sim 12 \mathrm{nb}$.

The $\tau$ plus missing transverse energy (MET) trigger requires $E_{M E T}>10 \mathrm{GeV}$ at L1. At L2 this is increased to $E_{M E T}>20 \mathrm{GeV}$ and a calorimeter cluster with an isolated track with $P_{T}>10$
$\mathrm{GeV} / c$ is required. This is followed by full event reconstruction at L3, requiring at least one $\tau$ candidate having seed track $P_{T}>4.5 \mathrm{GeV} / c$. The present trigger cross section is $5 \mathrm{nb}$.

\section{First Results}

The study of $Z \rightarrow \tau_{e} \tau_{h}$ is performed using $72 \mathrm{pb}^{-1}$ of data taken with the electron plus track trigger [3]. The track multiplicity associated with the $\tau_{h}$ candidate found in events passing selection cuts is shown in Fig. 1] We observe a clear $\tau$ signal over background levels even before requiring an opposite sign charge for the electron and the $\tau_{h}$. The mass distribution of the electron- $\tau_{h}$-MET system in the data is also found to be consistent with the $Z \rightarrow \tau \tau$ hypothesis.

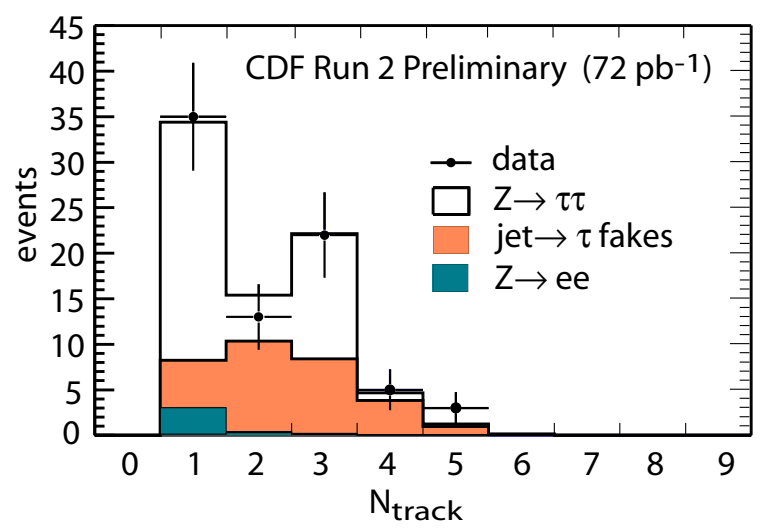

Figure 1. Track multiplicity for the $\tau$ candidate.

\section{REFERENCES}

1. The CDF II Detector: Technical Design Report, Fermilab-Pub-96/390-E (1996).

2. S. Holm et al., IEEE Trans. Nucl. Sci. 47:895(2000).

3. A. Safonov, in Proceedings of 38th Rencontres de Moriond on QCD and High-Energy Hadronic Interactions, 2003. FERMILABCONF-03/153-E. 